\title{
A Standard Model explanation for the MiniBooNE anomaly
}

\author{
Ara loannisian, ${ }^{a, b, *}$ Carlo Giunti $^{c}$ and Gioacchino Ranucci ${ }^{d}$ \\ ${ }^{a}$ Yerevan Physics Institute, \\ Alikhanian Brothers 2, 375036 Yerevan, Armenia \\ ${ }^{b}$ Institute for Theoretical Physics and Modeling, \\ 375036 Yerevan, Armenia \\ ${ }^{c}$ Istituto Nazionale di Fisica Nucleare (INFN), Sezione di Torino, \\ Via P. Giuria 1, I-10125 Torino, Italy \\ ${ }^{d}$ Istituto Nazionale di Fisica Nucleare (INFN), Sezione di Milano, \\ I-20133 Milano, Italy \\ E-mail: ara.ioannisyan@cern.ch, carlo.giunti@to.infn.it, \\ gioacchino.ranucci@mi .infn.it
}

We present the results of a new analysis of the data of the MiniBooNE experiment taking into account the additional background of photons. MiniBooNE normalises the rate of photon production to the measured $\pi^{0}$ production rate. We study neutral current (NC) neutrino-induced $\pi^{0} /$ photon production $\left(v_{\mu}+A \rightarrow v_{\mu}+1 \pi^{0} / \gamma+X\right)$ on carbon nucleus ( $\left.\mathrm{A}=12\right)$. Our conclusion is based on experimental data for photon-nucleus interactions from the A2 collaboration at the Mainz MAMI accelerator. We work in the approximation that decays of the intermediate states (non-resonant $\mathrm{N}, \Delta$ resonance, higher resonances) unaffected by its production channel, via photon or $\mathrm{Z}$ boson. $1 \pi^{0}+X$ production scales as $\mathrm{A}^{2 / 3}$, the surface area of the nucleus. Meanwhile the photons incoherently created in intermediate states decays will leave the nucleus, and that cross section will be proportional to the atomic number of the nucleus. We also took into account the coherent emission of photons. We show that the new photon background can explain part of the MiniBooNE low-energy excess, thus significantly lowering the number of unexplained MiniBooNE electron-like events from 5.1 $\sigma$ to $3.6 \sigma$.

40th International Conference on High Energy physics - ICHEP2020

July 28 - August 6, 2020

Prague, Czech Republic (virtual meeting)

\footnotetext{
${ }^{*}$ Speaker
} 


\section{Introduction}

The MiniBooNE experiment consists of a liquid-scintillator detector that uses mineral oil (methylene, $\mathrm{CH}_{2}$ ) to search for $\stackrel{(-)}{v_{\mu}} \rightarrow \stackrel{(-)}{v_{e}}$ oscillations. It reported a significant excess of low-energy (200-500 MeV) electron-like events in both neutrino and antineutrino runs [1-3]. This anomaly gave rise to speculations of new physics beyond the Standard Model [4].

It is well known that at these energies MiniBooNE cannot distinguish electrons/positrons from photons, and must therefore subtract the contribution due to photoproduction from their measured signal. They estimate the photoproduction rate from the NC process $v_{\mu}+\mathrm{CH}_{2} \rightarrow v_{\mu}+\gamma+\mathrm{X}$ using the number of detected $\pi^{0}$ s from $v_{\mu}+\mathrm{CH}_{2} \rightarrow v_{\mu}+\pi^{0}+X$.

We study neutral current (NC) neutrino-induced $\pi^{0} /$ photon production $\left(v_{\mu}+A \rightarrow v_{\mu}+1 \pi^{0} / \gamma+\right.$ $X)$ on carbon nucleus. Our results [5,6] are based on experimental data for photon-nucleus interactions from the A2 collaboration at the Mainz MAMI accelerator [7].

\section{The MiniBooNE single photon background}

In MiniBooNE the main source of single photons is $\Delta^{+/ 0} \rightarrow p / n+\gamma$ decay of $\Delta^{+/ 0}$ 's produced in neutral-current $v_{\mu}$ interactions with the mineral oil $\left(\mathrm{CH}_{2}\right)$ of the detector. The MiniBooNE estimated this background through the measurement of $\pi^{0}$ 's that are produced by the decay $\Delta^{+/ 0} \rightarrow p / n+\pi^{0}$, using the branching fractions [8]

$$
\begin{aligned}
& \operatorname{Br}\left(\Delta^{+/ 0} \rightarrow p / n+\gamma\right)=(6.0 \pm 0.5) \times 10^{-3}, \\
& \operatorname{Br}\left(\Delta^{+/ 0} \rightarrow p / n+\pi^{0}\right) \simeq 2 / 3 .
\end{aligned}
$$

Final state interactions (FSI) cause the absorption of a fraction of the $\pi^{0}$ 's in the carbon nucleus that was estimated to be about $37.5 \%$ by the MiniBooNE [2] . However, in Ref. [5, 6] it was noted that measurements of $\pi^{0}$ photoproduction on nuclei $[9,10]$ indicate that the fraction of $\pi^{0}$ 's that emerge from the nucleus and can be observed is given by

$$
\frac{N_{\pi^{0}}^{\mathrm{FSI}}}{N_{\pi^{0}}^{0}}=\frac{\sigma_{\mathrm{FSI}}\left(\gamma+{ }^{A} \mathcal{N} \rightarrow \pi^{0}+X\right)}{\sigma_{0}\left(\gamma+{ }^{A} \mathcal{N} \rightarrow \pi^{0}+X\right)} \simeq \frac{A^{2 / 3}}{A}=A^{-1 / 3},
$$

where $A$ is the mass number of the target nucleus ${ }^{A} \mathcal{N}, \sigma_{\mathrm{FSI}}$ denotes the measured cross section which includes final state interactions and $\sigma_{0}$ denotes the theoretical cross section without final state interactions. Since the nuclear radius scales approximately as $A^{1 / 3}$, the $A^{2 / 3}$ dependency of $\sigma_{\mathrm{FSI}}\left(\gamma+{ }^{A} \mathcal{N} \rightarrow \pi^{0}+X\right)$ indicates that only the nuclear surface contributes, whereas all the $\pi^{0}$ that are produced in the nuclear interior are absorbed [11]. However, the uncertainties of the $A^{2 / 3}$ scaling in Eq. (3) are not known.

We propose to consider the extreme total absorption of the $\pi^{0}$ that are produced in the nuclear interior as indicated by the $\pi^{0}$ photoproduction data $[9,10]$ as the possible cause of an increase of the estimated MiniBooNE single- $\gamma$ background that can explain at least part of the MiniBooNE low-energy excess. In other words, we consider Eq. (3) as an ansatz of the effects of FSI $\pi^{0}$ absorption in a nucleus that is motivated by the photoproduction data. The resulting estimate of the probability of $\pi^{0}$ escape from the ${ }^{12} \mathrm{C}$ nucleus is

$$
\widetilde{S}_{\mathrm{C}}\left(\pi^{0}\right) \simeq 12^{-1 / 3}=0.437
$$




\begin{tabular}{c|ccc|ccc}
\hline \hline & \multicolumn{3}{|c|}{$v$ mode } & \multicolumn{3}{c}{$\bar{v}$ mode } \\
$E_{v}^{\mathrm{QE}}(\mathrm{GeV})$ & {$[0.2,0.3]$} & {$[0.3,0.475]$} & {$[0.475,1.3]$} & {$[0.2,0.3]$} & {$[0.3,0.475]$} & {$[0.475,1.3]$} \\
\hline$f_{\text {coh }}^{\text {th }}$ & 0.09 & 0.13 & 0.06 & 0.16 & 0.16 & 0.07 \\
$f_{N^{*}}^{\text {th }}$ & 0.02 & 0.02 & 0.13 & 0.03 & 0.02 & 0.13 \\
$\widetilde{R} / R_{\mathrm{MB}}$ & 1.52 & 1.56 & 1.61 & 1.62 & 1.61 & 1.62 \\
\hline \hline
\end{tabular}

Table 1: Estimations of $f_{\text {coh }}^{\text {th }}$ and $f_{N^{*}}^{\text {th }}$ from Table 2 of Ref. [13] in three ranges of reconstructed neutrino energy $E_{v}^{\mathrm{QE}}$ in the $v$ and $\bar{v}$ modes of the MiniBooNE experiment, and the corresponding values of the enhancement factor $\widetilde{R} / R_{\mathrm{MB}}$ that we obtained considering $f_{N}^{\text {th }} \simeq 0.1$ [12] and our value (4) of the probability of $\pi^{0}$ escape from the ${ }^{12} \mathrm{C}$ nucleus.

that is smaller than that estimated by the MiniBooNE collaboration [2], $S_{\mathrm{C}}^{\mathrm{MB}}\left(\pi^{0}\right)=0.625$.

Moreover, the MiniBooNE assumed that "single gamma events are assumed to come entirely from $\Delta$ radiative decay" [2], neglecting the additional contributions to $\gamma$ production from coherent photon emission, incoherent production of higher mass resonances, and incoherent non-resonant nucleon production $[12,13]$. Taking into account these contributions and our value (4) of the probability of $\pi^{0}$ escape from the ${ }^{12} \mathrm{C}$ nucleus, the ratio of single-photon events to $\mathrm{NC} \pi^{0}$ events is given by

$$
\widetilde{R}=\frac{N_{\mathrm{H}}^{\mathrm{th}}(\Delta \rightarrow N \gamma)+N_{\mathrm{C}}^{\mathrm{th}}(\Delta \rightarrow N \gamma)+N_{\mathrm{coh}}^{\mathrm{th}}(\gamma)+N^{\mathrm{th}}\left(N^{*} \rightarrow N \gamma\right)+N^{\mathrm{th}}(N \rightarrow N \gamma)}{\widetilde{N}_{\mathrm{tot}}^{\mathrm{th}, \mathrm{obs}}\left(\pi^{0}\right)} .
$$

The contributions in the numerator are, respectively, the theoretically predicted numbers of single- $\gamma$ events due to $\Delta \rightarrow N \gamma$ in $\mathrm{H}, \Delta \rightarrow N \gamma$ in $\mathrm{C}$, coherent photon emission, incoherent production of higher mass resonances $\left(N^{*} \rightarrow N \gamma\right)$ and incoherent non-resonant nucleon production $(N \rightarrow N \gamma)$. The denominator is the theoretically predicted total number of observed $\pi^{0}$ events. Note that only the denominator of Eq. (5) depends on the probability of $\pi^{0}$ escape from the ${ }^{12} \mathrm{C}$ nucleus, because a larger escape probability implies a larger number of observed $\pi^{0}$ events. The tilde notation indicates that $\widetilde{N}_{\text {tot }}^{\text {thobs }}\left(\pi^{0}\right)$ corresponds to our value $\widetilde{S}_{\mathrm{C}}\left(\pi^{0}\right)$ in Eq. (4) of such probability.

We can write Eq. (5) as

$$
\begin{aligned}
\widetilde{R}= & \frac{N_{\mathrm{H}}^{\mathrm{th}}(\Delta \rightarrow N \gamma)+N_{\mathrm{C}}^{\mathrm{th}}(\Delta \rightarrow N \gamma)}{N_{\mathrm{tot}}^{\mathrm{th}, \mathrm{obs}}\left(\pi^{0}\right)}\left(\frac{N_{\mathrm{tot}}^{\mathrm{th}, \mathrm{obs}}\left(\pi^{0}\right)}{\widetilde{N}_{\mathrm{tot}}^{\mathrm{th}, \mathrm{obs}}\left(\pi^{0}\right)}\right) \\
& \times\left(1+f_{\mathrm{coh}}^{\mathrm{th}}+f_{N^{*}}^{\mathrm{th}}+f_{N}^{\mathrm{th}}\right),
\end{aligned}
$$

where $N_{\mathrm{tot}}^{\mathrm{th}, \mathrm{obs}}\left(\pi^{0}\right)$ is the total number of observed $\pi^{0}$ events estimated by the MiniBooNE collaboration using the probability of $\pi^{0}$ escape from the ${ }^{12} \mathrm{C}$ nucleus $S_{\mathrm{C}}^{\mathrm{MB}}\left(\pi^{0}\right)=0.625$ [2]. In Eq. (6)

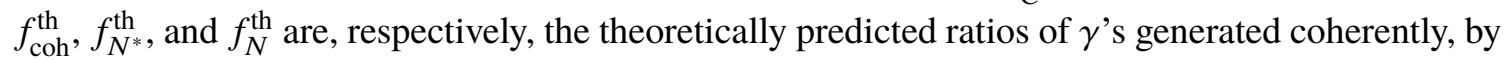
higher mass resonances, and non-resonant nucleon production with respect to those generated by $\Delta$ decay. In this way, we separated these contributions from the those generated by $\Delta$ decay that were considered by the MiniBooNE collaboration. 


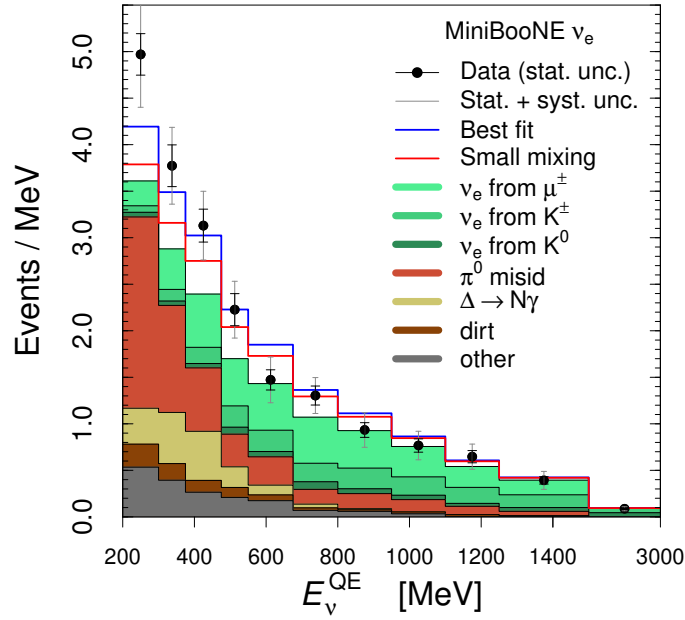

(a)

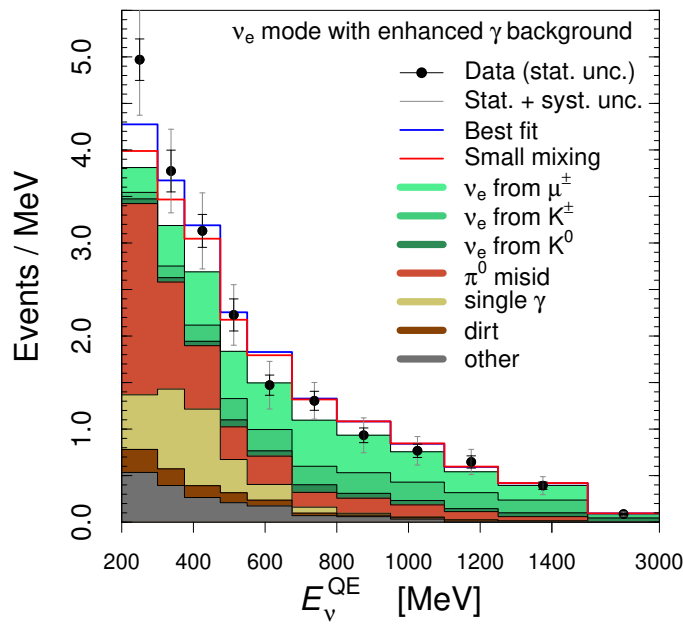

(c)

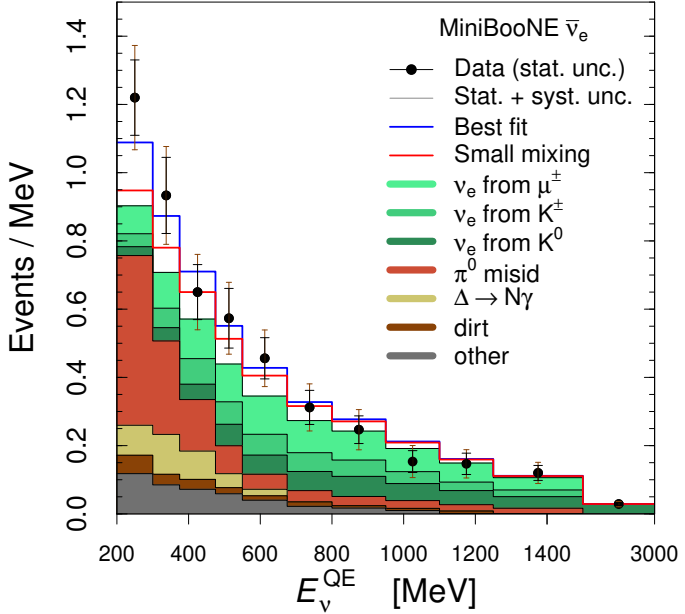

(b)

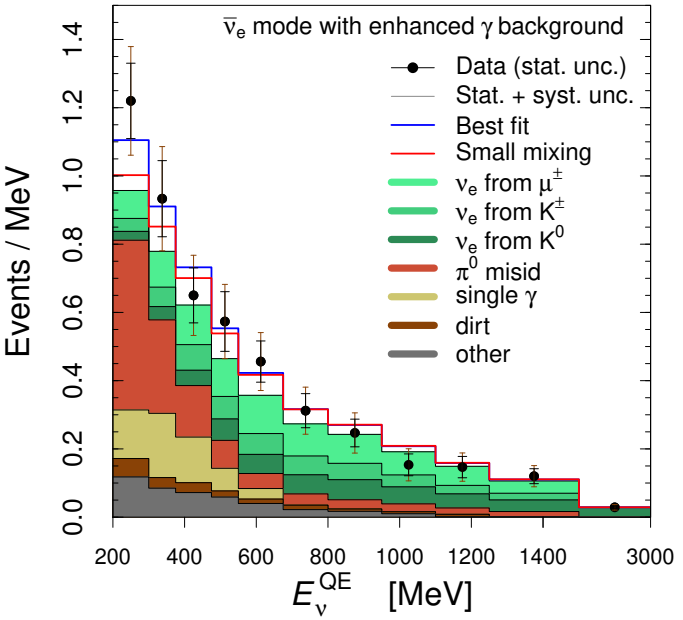

(d)

Figure 1: Comparison of a reproduction of the MiniBooNE electron-like event histograms in (a) neutrino and (b) antineutrino mode from Refs. [1-3] with our versions (c) and (d) obtained with the enhanced single- $\gamma$ background due to $A^{1 / 3} \pi^{0}$ FSI in ${ }^{12} \mathrm{C}$, coherent photon emission, incoherent production of higher mass resonances, and incoherent non-resonant nucleon production. The blue and red lines show, respectively, the expectations for neutrino oscillations corresponding to the best fit (almost maximal mixing) and the case of small mixing with $\sin ^{2} 2 \vartheta_{e \mu}=2.5 \times 10^{-3}$ and $\Delta m_{41}^{2}=0.8 \mathrm{eV}^{2}$.

The first fraction on the right-hand side of Eq. (6) is the ratio of single- $\gamma$ events to NC $\pi^{0}$ events estimated by the MiniBooNE collaboration:

$$
R_{\mathrm{MB}}=\frac{N_{\mathrm{H}}^{\mathrm{th}}(\Delta \rightarrow N \gamma)+N_{\mathrm{C}}^{\mathrm{th}}(\Delta \rightarrow N \gamma)}{N_{\mathrm{tot}}^{\mathrm{th}, \mathrm{obs}}\left(\pi^{0}\right)}=0.0091 .
$$

The second fraction on the right-hand side of Eq. (6) can be calculated by writing it as

$$
\frac{N_{\mathrm{tot}}^{\mathrm{th}, \mathrm{obs}}\left(\pi^{0}\right)}{\widetilde{N}_{\mathrm{tot}}^{\mathrm{th}, \mathrm{obs}}\left(\pi^{0}\right)}=\frac{N_{\mathrm{abs}}^{\mathrm{th}, \mathrm{obs}}\left(\pi^{0}\right)+N_{\text {noabs }}^{\mathrm{th}, \mathrm{obs}}\left(\pi^{0}\right)}{\widetilde{N}_{\mathrm{abs}}^{\mathrm{th}, \mathrm{obs}}\left(\pi^{0}\right)+N_{\text {noabs }}^{\text {th,obs }}\left(\pi^{0}\right)},
$$


where $N_{\text {abs }}^{\text {thobs }}\left(\pi^{0}\right)$ and $\widetilde{N}_{\text {abs }}^{\text {th,obs }}\left(\pi^{0}\right)$ are the theoretically predicted numbers of observed $\pi^{0}$ produced in processes with absorption of $\pi^{0}$ in the C nucleus, whereas $N_{\text {noabs }}^{\text {th,obs }}\left(\pi^{0}\right)$ is the theoretically predicted numbers of observed $\pi^{0}$ produced in processes without absorption of $\pi^{0}$ in the $\mathrm{C}$ nucleus. Note that only $N_{\text {abs }}^{\text {thobs }}\left(\pi^{0}\right)$ and $\widetilde{N}_{\text {abs }}^{\text {thobs }}\left(\pi^{0}\right)$ depend on the probability of $\pi^{0}$ escape from the ${ }^{12} \mathrm{C}$ nucleus and are given by $N_{\text {abs }}^{\text {th,obs }}\left(\pi^{0}\right)=N_{\text {abs }}^{\text {th,prod }}\left(\pi^{0}\right) S_{\mathrm{C}}^{\text {th }}\left(\pi^{0}\right)$ and $\widetilde{N}_{\text {abs }}^{\text {th,obs }}\left(\pi^{0}\right)=N_{\text {abs }}^{\text {th,prod }}\left(\pi^{0}\right) \widetilde{S}_{\mathrm{C}}^{\text {th }}\left(\pi^{0}\right)$. Therefore, we can write Eq. (8) as

$$
\frac{N_{\text {tot }}^{\text {th,obs }}\left(\pi^{0}\right)}{\widetilde{N}_{\text {tot }}^{\text {th,obs }}\left(\pi^{0}\right)}=\frac{1+\frac{N_{\text {noabs }}^{\text {thoobs }}\left(\pi^{0}\right)}{N_{\text {abs }}^{\text {th,obs }}\left(\pi^{0}\right)}}{\frac{\widetilde{S}_{\mathrm{C}}^{\text {th }}\left(\pi^{0}\right)}{S_{\mathrm{C}}^{\text {th }}\left(\pi^{0}\right)}+\frac{N_{\text {noabs }}^{\text {th,obs }}\left(\pi^{0}\right)}{N_{\text {abs }}^{\text {th,obs }}\left(\pi^{0}\right)}}
$$

We obtained the value of $N_{\text {noabs }}^{\text {th,obs }}\left(\pi^{0}\right) / N_{\mathrm{abs}}^{\mathrm{th}, \mathrm{obs}}\left(\pi^{0}\right)$ from the contributions to the MiniBooNE $\pi^{0}$ event sample given in Ref. [2]:

$$
\frac{N_{\text {noabs }}^{\text {th,obs }}\left(\pi^{0}\right)}{N_{\mathrm{abs}}^{\text {th,obs }}\left(\pi^{0}\right)} \simeq 0.496 .
$$

The resulting enhancement factor of the MiniBooNE single- $\gamma$ background is

$$
\frac{\widetilde{R}}{R_{\mathrm{MB}}} \simeq 1.25\left(1+f_{\mathrm{coh}}^{\mathrm{th}}+f_{N^{*}}^{\mathrm{th}}+f_{N}^{\mathrm{th}}\right)
$$

The authors of Ref. [13] calculated the number of single photon events from neutral current interactions at MiniBooNE. From their Table 2 we obtained the estimates of $f_{\text {coh }}^{\text {th }}$ and $f_{N^{*}}^{\text {th }}$ in Table 1 , considering three ranges of $E_{v}^{\mathrm{QE}}$ in the $v$ and $\bar{v}$ modes of the MiniBooNE experiment. For $f_{N}^{\text {th }}$ we considered the $10 \%$ value estimated in Ref. [12]. Note that we did not use the absolute values of the events calculated in Refs. [12,13], that are in agreement with the MiniBooNE estimates, and hence in disagreement with our estimations. We used only the relative values of the $\gamma$ 's generated coherently, by higher mass resonances, and non-resonant nucleon production, whose estimation can be considered more accurate.

As shown in Table 1, we find an enhancement $\widetilde{R} / R_{\mathrm{MB}}$ of the single- $\gamma$ background in MiniBooNE by a factor between 1.52 and 1.62 depending on the energy range and neutrino or antineutrino mode of the MiniBooNE experiment. This increase of the single- $\gamma$ background can explain in part the low-energy MiniBooNE excess, because its largest contribution occur in the lowest energy bins, as one can see from Figures 1(a) and 1(b) that reproduce the MiniBooNE event histograms in neutrino and antineutrino mode in Refs. [1-3].

Figure 1 shows a comparison of the standard MiniBooNE event histograms (Figures 1(a) and 1(b)) with those obtained with our reevaluation of the single- $\gamma$ background (Figures 1(c) and 1(d)). One can see that in the reproductions 1(a) and 1(b) of the original MiniBooNE histograms the low-energy bins show a large excess with respect to the background prediction. The excess is significantly reduced with our enhanced single- $\gamma$ background. Only the first energy bin remains with a large visible excess. 


\section{Summary}

We have shown that a reassessment of the single- $\gamma$ background from $\Delta^{+/ 0}$ decay in the MiniBooNE experiment taking into account the effect of $A^{1 / 3} \pi^{0}$ FSI proposed in Ref. [5] and additional contributions to the single- $\gamma$ background can explain in part the low-energy MiniBooNE excess. In absence of physics beyond the standard three-neutrino mixing, our enhanced single- $\gamma$ background leads to a better fit of the data, with a goodness-of-fit of $2 \%$, with respect to the standard analysis, that has a goodness-of-fit of $0.02 \%$. The statistical significance of the indication in favour of $v_{\mu} \rightarrow v_{e}$ oscillation decreases from $5.1 \sigma$ to $3.6 \sigma$.

\section{References}

[1] A. A. Aguilar-Arevalo et al. [MiniBooNE Collaboration], Phys. Rev. Lett. 121, no. 22, 221801 (2018)

[2] A. A. Aguilar-Arevalo et al. [MiniBooNE], [arXiv:2006.16883 [hep-ex]].

[3] A. A. Aguilar-Arevalo et al. [MiniBooNE Collaboration], Phys. Rev. Lett. 110, 161801 (2013)

[4] C. Giunti and T. Lasserre, Ann. Rev. Nucl. Part. Sci. 69, 163 (2019)

[5] A. Ioannisian, [arXiv:1909.08571 [hep-ph]].

[6] C. Giunti, A. Ioannisian and G. Ranucci, JHEP 11 (2020), 146

[7] https://wwwa2.kph.uni-mainz.de/

[8] M. Tanabashi et al. [Particle Data Group], Phys. Rev. D 98, no. 3, 030001 (2018).

[9] B. Krusche, Prog. Part. Nucl. Phys. 55, 46 (2005)

[10] B. Krusche, J. Lehr, J. Ahrens, J. R. M. Annand, R. Beck, F. Bloch, L. S. Fog, D. Hornidge, S. Janssen and M. Kotulla, et al. Eur. Phys. J. A 22 (2004), 277-291

[11] B. Krusche, J. Lehr, F. Bloch, M. Kotulla, V. Metag, U. Mosel and S. Schadmand, Eur. Phys. J. A 22 (2004), 347-351

[12] X. Zhang and B. D. Serot, Phys. Lett. B 719 (2013), 409-414

[13] E. Wang, L. Alvarez-Ruso and J. Nieves, Phys. Lett. B 740 (2015), 16-22

[14] Similar value of $\pi^{0}$ escape factor from carbon nucleus was reported in section 3.3 in T. Leitner, O. Buss, U. Mosel and L. Alvarez-Ruso, PoS NUFACT 08, 009 (2008) 